\title{
Búsqueda de aneurisma de aorta abdominal por cardiólogos
}

\author{
Gabriel Perea* \\ Adrián D’Ovidio \\ Mariana Corneli \\ *Instituto Cardiovascular de Buenos Aires. Buenos Aires. Argentina
}

Correspondencia

Gabriel Perea

e-mail: operea@icba.com.ar

Recibido: 16/01/2020

Aceptado: 16/01/2020

En línea: 02/03/2020

Citar como: Perea G, Corneli M, D’Ovidio A. Búsqueda de aneurisma de aorta abdominal por cardiólogos. Rev Ecocar Pract (RETIC). 2020 (Mar); 3 (1): 66-68. doi: 10.37615/retic.v3n1a18.

Cite this as: JPerea G, Corneli M, D'Ovidio A. Screening of abdominal aortic aneurysm by cardiologists. Rev Ecocar Pract (RETIC). 2020 (Mar); 3 (1): 66-68. doi: 10.37615/retic.v3n1a18

\section{Palabras clave \\ $\triangleright$ Aneurisma \\ $\triangleright$ Aorta abdominal \\ $\triangleright$ Ecografía}

\begin{tabular}{l}
\hline Keywords \\
\hline Aneurysm \\
$\triangleright$ Abdominal aorta \\
$\triangleright$ Echography \\
\end{tabular}

Introducción

La ecografía en sus distintas modalidades (modo bidimensional y Doppler) permite evaluar distintas estructuras del abdomen y así poder distinguir lo normal de lo anormal. Los cardiólogos aprenden a explorar la aorta abdominal (AA) en el momento en que se familiarizan con las vistas subxifoideas de la ecocardiografía; fundamentalmente cuando es necesario definir el grado de severidad de la insuficiencia valvular aórtica al evaluar el flujo reverso a dicho nivel o cuando se busca un flujo anormal por coartación de la aorta.

\section{Cómo evaluar la aorta abdominal}

En muchas oportunidades será posible cuantificar las dimensiones de la aorta abdominal desde el abordaje subxifoideo al realizar una ecocardiografía Doppler color y así buscar la presencia de un aneurisma de aorta abdominal (AAA).

Las mediciones de la aorta abdominal se realizan en cortes transversos perpendiculares al eje del vaso. Las medidas se limitan desde el borde externo (adventicia) de la pared, privilegiando la medición anteroposterior. Esta medición se recomienda realizar a nivel proximal, medio y distal en relación al origen de las arterias renales. De esta forma ante la pregunta de si es posible diagnosticar un
AAA en un examen ecocardiográfico utilizando un transductor cardiológico, la respuesta es sí.

\section{Aneurisma de aorta abdominal}

Se define aorta abdominal normal por tamaño cuando presenta un diámetro máximo de $20 \mathrm{~mm}^{(1-2)}$, aunque es importante considerar variabilidades asociadas a la superficie corporal. Cuando el diámetro de la aorta abdominal es 1,5 veces mayor al valor de un segmento adyacente o presenta un valor absoluto mayor a $30 \mathrm{~mm}$ se considera AAA.

La prevalencia documentada de AAA es de 1,3\% para hombres hasta los 54 años, incrementándose a 12,5\% para varones de 75 a 84 años ${ }^{(1)}$. Por eso es importante evaluar fundamentalmente a pacientes mayores a 65 años. Más aun en aquellos con antecedentes familiares de AAA o tabaquismo.

Los pacientes con AAA suelen estar asintomáticos, por eso es importante tener a mano una simple herramienta que permita su identificación. Diversos estudios han demostrado los beneficios de su detección temprana. El estudio Multicentre Aneurysm Screening Study (MASS) evidencio que en hombres $>65$ años la búsqueda de AAA reducía la mortalidad; sin olvidar además que el tamaño del aneurisma es un predictor de rotura. En AAA $<50 \mathrm{~mm}$ el riesgo de ruptura informado es de $20 \%, 40 \%$ hasta $60 \mathrm{~mm}$ y $>50 \%$ cuando el aneurisma supera los $70 \mathrm{~mm}^{(3)}$. 
Otros registros han arrojado resultados similares como el estudio UK trial donde se comparó la intervención temprana frente a la conducta expectante en aneurismas aórticos infrarrenales que tenían diámetros de 40-54 mm ${ }^{(4)}$.

\section{Técnica para la realización del estudio}

Es recomendable iniciar el examen con el transductor ubicado en posición subcostal. La AA está localizada, longitudinalmente, medial y ligeramente a la izquierda de la columna vertebral a nivel lumbar. Hay que tratar de generar la menor compresión posible en el abdomen para evitar la contractura de los músculos abdominales por parte del paciente.

La AA debe estudiarse progresivamente en corte transversal y, a continuación, de forma longitudinal desde el diafragma hasta la bifurcación aortoilíaca. Las medidas se realizan en una vista transversal, con el haz del transductor perpendicular al eje mayor de la $\mathrm{AA}^{(1)}$

Los diámetros anteroposterior y transversal suelen ser iguales. Si no es posible obtener una sección circular de la aorta (en caso de dilatación y/o sinuosidad), se puede calcular el diámetro medio de la elipse o tratar de medir el diámetro de la AA en una buena vista longitudinal, siempre que el diámetro sea perpendicular al eje de la AA (Figura 1). Los diámetros deben medirse de adventicia a adventicia. La medición del diámetro anteroposterior es más exacta que el diámetro transverso, ya que la perpendicularidad del ultrasonido confiere una vista más precisa (Figura 2 y Figura 3$)^{(5)}$

Para evaluar los flujos tanto con Doppler color como con pulsado se recomienda usar un volumen de muestra similar a la usada en ecocardiografía, excepto que se usa una corrección angular de $60^{\circ}$ en forma ideal, teniendo en cuenta que la dirección del flujo debe ser lo más paralelo posible al volumen de muestra(5)

Hay que recordar que en presencia de un AAA el flujo suele ser ectásico, por lo que será necesario utilizar valores de frecuencia de repetición de pulsos (PRF) bajos para valorar adecuadamente los flujos tanto con Doppler color como Doppler espectral.

\section{Estudio por imagen}

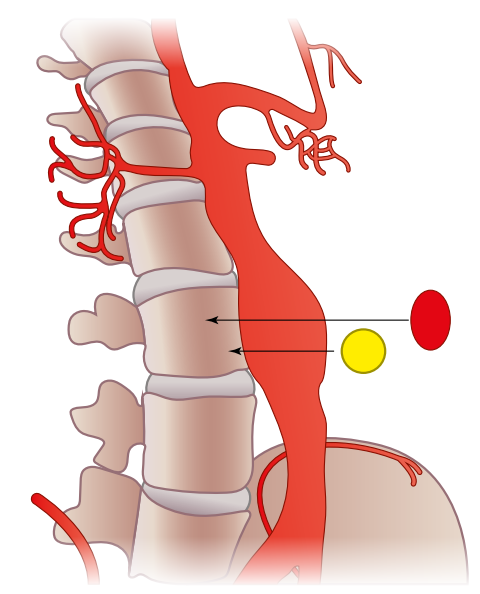

Figura 1. Corte perpendicular (amarillo) y oblicuo (rojo) de la aorta abdominal. Se recomienda la búsqueda de la perpendicularidad para obtener imagen transversa circular y no oval

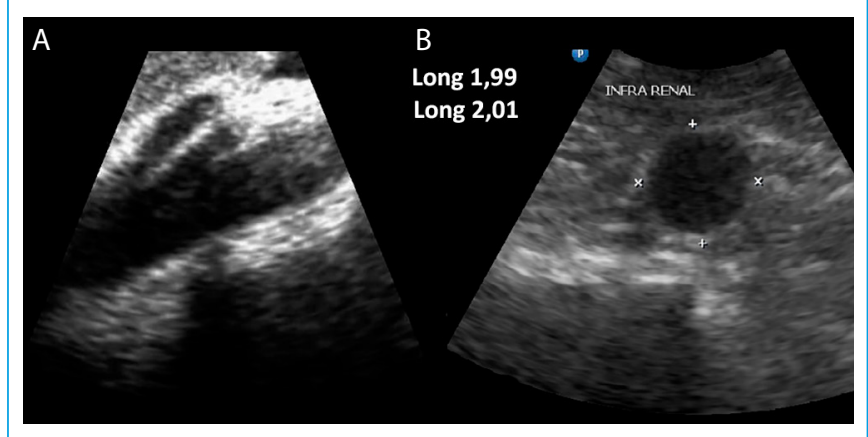

Figura 2. Imágenes obtenidas con el transductor para estudio de ecocardiografía. A: vista longitudinal donde se identifica el origen de arteria mesentérica superior; B: vista transversal con mediciones a nivel de la aorta infrarrenal

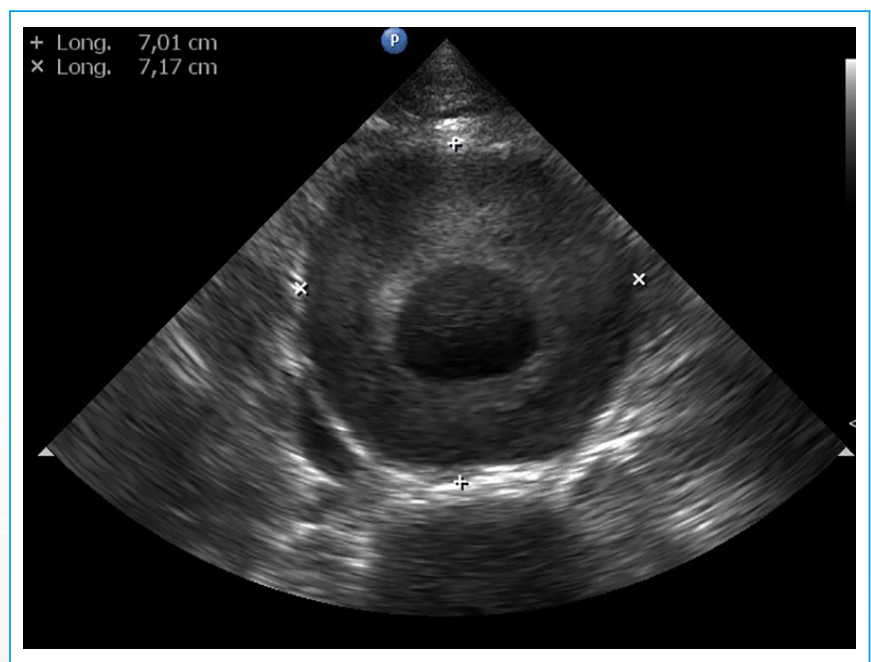

Figura 3. Aneurisma de aorta abdominal medido en diámetros anteroposterior y trasverso. La heterogeneidad de la ecogenicidad mural corresponde a trombo mural y el lumen se observa anecoico

\section{Conclusión}

Dada la variabilidad de dimensiones descritas en distintas poblaciones y la escasez de estudios que representan a la población latinoamericana desde la SISIAC se iniciará un registro con acceso on line para recabar datos en cuanto a las medidas obtenidas en la población de Latinoamérica de la aorta abdominal. Dicho registro incluirá mediciones de la aorta abdominal a nivel proximal, medio y distal realizadas por médicos cardiólogos y especialidades afines que quieran participar, así como la posibilidad de obtener cifras de la realidad latinoamericana sobre el aneurisma de aorta abdominal.

\section{Ideas para recordar}

- Tratar de lograr vistas trasversas perpendiculares al eje de la aorta para su medición. Medir incluyendo todas las paredes de aorta abdominal (de adventicia a adventicia)

- Medir por arriba o proximal respecto de la arteria mesentérica superior o arterias renales (suprarrenal) por debajo de las mismas a distancia equidistante de la bifurcación ilíaca (infrarrenal) y previo a la bifurcación, algunas veces el diámetros yuxtarrenal (entre los ostium de las renales) o a nivel de la arteria mesentérica suele ser muy útil. 


\section{Bibliografía}

1. Ricci MA, Kleeman M, Case T, Pilcher DB. Normal aortic diameter by ultrasound. J Vasc Technol 1995; 19: 17-19.

2. Lederle FA, Jhonson GR, Wilson SE, et al. Relationship of age, gender, race and body size to infrarenal aortic diamenter. J Vasc Surg 1997; 25: 595-601.

3. The Multicentre Aneurysm Screening Study (MASS) into the effect of abdominal aortic aneurysm screening on mortality in men: a randomized controlled trial. Lancet 2002; 360: 1531-1539.
4. Brown LC, Powell JT. UK Small Aneurysm Trial Participants. Risk factors for aneurysm rupture in patients kept under ultrasound surveillance. Ann Surg 1999; 230: 289-296.

5. Sprynger M, Rigo F, Moonen M, et al. Focus on echovascular imaging assessment of arterial disease: complement to the ESC guidelines (PARTIM1) in collaboration with the Working Group on Aorta and Peripheral Vascular Diseases. European Heart Journal - Cardiovascular Imaging 2018; 0: 1-27. 